\title{
PARABOLIC EQUATIONS ASSOCIATED WITH THE NUMBER OPERATOR
}

\author{
BY \\ M. ANN PIECH(1)
}

\begin{abstract}
We study existence and uniqueness of solutions of the Cauchy problem for $\dot{u}=N u$ where $N$ is the number operator on abstract Wiener space.
\end{abstract}

1. Introduction. Recent work in mathematical quantum field theory and in integration over Banach manifolds indicates that in an infinite-dimensional setting a suitable formulation of the differential operator $N=\Delta-x \cdot \nabla$ may play a more fundamental role than the Laplacian $\Delta$. In order to study $N$ on manifolds it is appropriate to study a version of $N$ with $x$-dependent coefficients. An appropriate infinite-dimensional setting is that of an abstract Wiener space, for it is here that we have a useful integration theory. In this paper we study properties of the fundamental solution for $\dot{u}=N u$. We are primarily interested in properties which will enable future use of this fundamental solution as a parametrix for a fundamental solution of $\dot{u}=N_{x} u$, where $N_{x}$ is a version of $N$ with variable coefficients.

L. Gross [5] has made a study of the infinite-dimensional Laplacian on an abstract Wiener space $(H, B, i) . H$ is a given real separable Hilbert space with inner product $(\cdot, \cdot)$ and norm $|\cdot| ; B$ is the completion of $H$ with respect to a given measurable norm $\|\cdot\|$ on $H$; and $i$ is the canonical embedding of $H$ in $B$. Integration over $B$ is performed with respect to Wiener measure $p_{t}$ with variance parameter $t>0$. For a real-valued function $f$ defined on $H, \Delta f(x)$ is defined as tr $D^{2} f(x)$, the trace of the second Fréchet derivative of $f$ evaluated at $x$. Here $D^{2} f(x)$ is regarded as a bounded linear operator on $H$ via the canonical isomorphism between $H$ and its dual space $H^{*}$. In order to obtain regularity results concerning $\Delta$, it is desirable to define $\Delta f(x)$ for $x$ ranging over $B$. This is accomplished in the following manner. For a fixed $x$ in $B$, define $g: H \rightarrow R$ by $g(h) \equiv f(x+h)$. The first and second Fréchet derivatives of $f$ at $x$ are, respectively, $D f(x) \equiv D g(0) \in H^{*} \approx H$ and $D^{2} f(x) \equiv D^{2} g(0) \in L\left(H^{*}, H\right)$ $\approx L(H, H)$. Then $\Delta f(x) \equiv \operatorname{tr} D^{2} f(x)$ whenever $D^{2} f(x)$ exists and is of trace class.

In finite dimensions $x \cdot \nabla f(x)$ is the directional derivative of $f$ in the direction of $x$, and so for any $x$ in $B$ we will define

Received by the editors August 8, 1973 and, in revised form, October 25, 1973.

AMS (MOS) subject classifications (1970). Primary 35K15; Secondary 60J60, $28 \mathrm{~A} 40$.

Key words and phrases. Parabolic equations, number operator, Ornstein-Uhlenbeck process, abstract Wiener space.

(1) This work was supported by NSF grant PO 28934. 


$$
N f(x) \equiv \Delta f(x)-\lim _{s \rightarrow 0} \frac{f((1+s) x)-f(x)}{s}
$$

whenever the right side of (1) exists. We will call $N$ the number operator, since (up to a scaling factor) it may be obtained as the image of the number of particles operator on Fock space $\mathscr{F}$ under an isomorphism which sends $\mathscr{F}$ to $L^{2}\left(p_{1}\right)$ (see Segal [15], Nelson [12]).

From the Fock space theory we find that, on a suitable domain in $L^{2}\left(p_{1}\right), N$ is essentially selfadjoint. The spectrum of $\bar{N}$ is discrete and nonpositive, and the eigenfunctions are obtained from Hermite polynomials. For each $t>0, e^{t \bar{N}}$ is a contraction operator in $L^{2}$, and, given any $\lambda>2, e^{t \bar{N}}$ is a contraction from $L^{2}$ to $L^{\lambda}$ for all $t$ sufficiently large.

The use of $N$ as an infinite-dimensional Laplacian has been motivated by Umemura [17]. This is discussed by McKean [9] as a consequence of viewing Wiener measure as the uniform distribution on an infinite-dimensional spherical surface of radius $\infty^{1 / 2}$.

Goodman [1] has developed a divergence theorem on abstract Wiener spaces. When a vector field $F$ is of the form $\nabla g$ and when Wiener measures are used, it can be seen from Corollary 2.1 of [1] that a natural definition of (Div $F)(x)$ is $\mathrm{Ng}(x)$. Goodman's divergence theorem then gives (formally) $\int_{V} \operatorname{Div} F=\int_{\partial V} F$ - $n$, and so $N$ takes over another desirable function for an infinite-dimensional Laplacian.

In this paper we will study the Cauchy problem for the equation $N u=u_{t}$. When $H=R^{n}$, then $B$ also equals $R^{n}$ and Wiener measure becomes Gauss measure. The solution semigroup $\left\{T_{t}: t>0\right\}$ for $N u=u_{t}$ is given by

$$
T_{t} f(x)=\left(2 \pi\left(1-e^{-2 t}\right)\right)^{-n / 2} \int_{R^{n}} f\left(e^{-t} x-y\right) \exp \left(-|y|^{2} / 2\left(1-e^{-2 t}\right)\right) d y .
$$

These transition measures may be interpreted on abstract Wiener space, and we will show that they provide a fundamental solution for the Cauchy problem. Due to symmetry of $N$ we will be able to show uniqueness of solutions. We also look at the fundamental solution when the second order coefficients of $N$ are constant but not $I$.

2. Solution of $N u=u_{t}$. In this section we will concern ourselves with solutions of the parabolic problem

$$
\begin{aligned}
N g(x, t) & =\frac{\partial}{\partial t} g(x, t) \quad(x \in B, t>0) \\
\lim _{t \downarrow 0} g(x, t) & =f(x) \quad \text { uniformly for } x \in B .
\end{aligned}
$$

We follow the convention that for a function of space $(x)$ and time $(t), N, D$ and $D^{2}$ will operate only with respect to the space variables. A function $f$ on $B$ is uniformly Lip 1 if there is a constant $C$ such that $|f(x)-f(y)| \leq C\|x-y\|$ for all $x$ and $y$ in $B$. We will establish the following 
Theorem 1. Assume $f$ is bounded and uniformly Lip 1 on B. Then

$$
g(x, t) \equiv p_{1-e^{-2 t}} f\left(e^{-t} x\right)
$$

satisfies problem (2). Moreover

(a) $D g(x, t)$ is in $B^{*}$ for each $x$ and $t$, and so

$$
\lim _{s \rightarrow 0} \frac{g((1+s) x, t)-g(x, t)}{s}=\langle x, D g(x, t)\rangle
$$

where $\langle$,$\rangle denotes the B-B^{*}$ pairing. This limit exists uniformly on bounded subsets of $B$. Moreover the map $(x, t) \rightarrow\langle x, D g(x, t)\rangle$ is uniformly continuous on $S \times[\tau, \infty)$ where $S$ is any bounded subset of $B$ and $\tau$ is $>0$.

(b) For each $\tau>0$, the map $(x, t) \rightarrow D^{2} g(x, t)$ is uniformly continuous on $B \times[\tau, \infty)$ into the Banach space of trace class operators on $H$.

(c) For each $t>0, \partial g(x, t) / \partial t$ exists uniformly for $x$ in any bounded subset of $B$.

(d) $|g(x, t)| \leq\|f\|_{\infty}$.

$\left\|D^{2} g(x, t)\right\|_{\mathrm{tr}} \leq c_{1} t^{-1 / 2} e^{-t}$ for some constant $c_{1}$ depending only on the B-norm.

$|N g(x, t)| \leq c_{2} e^{-t}\left(t^{-1 / 2}+\|x\|\right)$ for some constant $c_{2}$ depending only on the $B$ norm and on the Lip 1 constant of $f$.

Lemma 2.1 (Gross [5, Theorem 3]). Assume $f$ is bounded and uniformly Lip 1 on B. Then $h(x, t) \equiv p_{t} f(x)$ satisfies

$$
\begin{aligned}
\frac{1}{2} \operatorname{tr}\left[D^{2} h(x, t)\right] & =\frac{\partial}{\partial t} h(x, t) \quad(x \in B, t>0) \\
\lim _{t>0} h(x, t) & =f(x) \quad \text { uniformly for } x \text { in } B .
\end{aligned}
$$

Moreover

(a) $h(x, t)$ is jointly uniformly continuous on $B \times[0, \infty)$.

(b) For each $\tau>0, \partial h / \partial t$ is bounded and uniformly continuous on $B \times[\tau, \infty)$, and $\partial h / \partial t$ exists uniformly in $x$ for each $t>0$.

(c) For each $\tau>0$, the map $(x, t) \rightarrow D^{2} h(x, t)$ is uniformly continuous on $B \times[\tau, \infty)$ into the Banach space of trace class operators on $H$.

The next lemma, while not necessary for our proof of Theorem 1, is presented to illustrate what portion of that theorem may be obtained from Lemma 2.1 via a straightforward chain rule computation.

Lemma 2.2. Assume $f$ is bounded and uniformly Lip 1 on $B$. Then, for $x$ in $H$, $g(x, t)$ satisfies problem (2) when restricted to $H$.

Proof. Set $F(u, v)=p_{v} f(u)$ where $u=u(x, t)=e^{-t} x \in H$ and $v=v(t)=1$ $-e^{-2 t}$. Set $(u, v)=w . w \in H \times R$ and so Lemma 2.1 ensures the existence of $d F / d w$, since the $u$ and $v$ partials exist and are continuous. The lemma follows on applying the chain rule. 
Remark 2.3. For $x$ in $H$ and $y$ in $B^{*}$, when we make the identification $H \subseteq B$ and $B^{*} \subseteq H^{*} \approx H$, we obtain $\langle x, y\rangle=(x, y)$.

Remark 2.4. When $x \in B \backslash H$, for a chain rule calculation to work we would have to have the existence of the $B$-derivative of $p_{t} f(x)$. Since $p_{t} f(x)$ is generally not $B$-differentiable, the next best thing we could hope for would be that $p_{t} f(x)$ have directional derivatives in all directions of $B$. This is, in fact, the case as the following lemmas show.

Lemma 2.5 (see Goodman [2]). Assume $f$ is bounded and uniformly Lip 1 on $B$. Then $D p_{t} f(x) \in B^{*}$, and the map $(y, x) \rightarrow\left\langle y, D p_{t} f(x)\right\rangle$ is uniformly continuous on $S \times B$, where $S$ is any bounded subset of $B$.

Proof. If $|f(x)-f(y)| \leq C\|x-y\|$ for all $x$ and $y$ in $B$, then it follows from the definition of $p_{t} f(x)$ that $\left|p_{t} f(x)-p_{t} f(y)\right| \leq C\|x-y\|$. By Proposition 9 of [5], $D p_{t} f(x)$ exists and is given by the formula

$$
\left(D p_{t} f(x), h\right)=t^{-1} \int_{B} f(x+y)(h, y) p_{t}(d y)
$$

for $h$ in $H$. Here $y \rightarrow(h, y)$ is the measurable function on $B$ determined by the tame function $y \rightarrow(h, y)$ defined for all $y$ in $H$ [3], [4]. Now for $h$ in $H$,

$$
\begin{aligned}
\left\langle h, D p_{t} f(x)\right\rangle \mid & =\left|\lim _{s \rightarrow 0} s^{-1}\left[p_{t} f(x+s h)-p_{t} f(x)\right]\right| \\
& \leq \limsup _{s \rightarrow 0}|s|^{-1}\left|p_{t} f(x+s h)-p_{t} f(x)\right| \\
& \leq \limsup _{s \rightarrow 0} C \frac{\|s h\|}{|s|}=C\|h\| .
\end{aligned}
$$

In fact, this calculation shows that for any bounded uniformly Lip $1 \mathrm{H}$ differentiable function on $B$, the $H$ derivative is in $B^{*}$, with $B^{*}$ norm dominated by the Lip 1 constant of the function.

For $h$ in $H,(3)$ gives

$$
\begin{aligned}
\mid\left\langle h, D p_{t}\right. & \left.f\left(x_{1}\right)-D p_{t} f\left(x_{2}\right)\right\rangle \mid \\
& \leq t^{-1}\left\{\int_{B}(h, y)^{2} p_{t}(d y)\right\}^{1 / 2} \cdot\left\{\int_{B}\left|f\left(x_{1}+y\right)-f\left(x_{2}+y\right)\right|^{2} p_{t}(d y)\right\}^{1 / 2} \\
& \leq t^{-1} \cdot t^{1 / 2}|h| \cdot C\left\|x_{1}-x_{2}\right\|,
\end{aligned}
$$

and so for fixed $h$ and $t>0, x \rightarrow\left\langle h, D p_{t} f(x)\right\rangle$ is uniformly continuous on $B$. For $y$ in $B\left|\left\langle h, D p_{t} f(x)\right\rangle-\left\langle y, D p_{t} f(x)\right\rangle\right| \leq C\|h-y\|$ by (4), and so for fixed $y$ and $t$, $x \rightarrow\left\langle y, D p_{t} f(x)\right\rangle$ is the uniform limit of uniformly continuous functions and so is uniformly continuous on $B$. Continuity of $(y, x) \rightarrow\left\langle y, D p_{t} f(x)\right\rangle$ on $B \times B$ is now a consequence of the bilinearity of the $B-B^{*}$ pairing. In fact, if $S$ is a bounded subset of $B$, then $\left\langle y, D p_{t} f(x)\right\rangle$ is uniformly continuous on $S \times B$ (for fixed $t>0$ ). 
Lemma 2.6. $\lim _{s \rightarrow 0} s^{-1}\left[p_{t} f(x+s y)-p_{t} f(x)\right]=\left\langle y, D p_{t} f(x)\right\rangle$ for all $y$ in B. For fixed $t>0$, the limit exists uniformly for $x$ in $B$ and $y$ in $a$ bounded subset of $B$.

Proof. For $y$ in $H$, it is a consequence of the existence of $D p_{t} f(x)$ that

$$
\left.\frac{\partial}{\partial s} p_{t} f(x+s y)\right|_{s=0}=\lim _{s \rightarrow 0} s^{-1}\left[p_{t} f(x+s y)-p_{t} f(x)\right]=\left\langle y, D p_{t} f(x)\right\rangle .
$$

This implies that for $y$ in $H$

$$
p_{t} f(x+s y)-p_{t} f(x)=\int_{0}^{s}\left\langle y, D p_{t} f(x+l y)\right\rangle d l .
$$

By Lemma 2.5, each side of (6) is continuous in $y$, for $y$ in $B$. Hence (6) holds for all $y$ in $B$. By the mean value theorem, there is an $l_{0}$ in $[0, s]$ such that

$$
\begin{aligned}
& \left|s^{-1}\left[p_{t} f(x+s y)-p_{t} f(x)\right]-\left\langle y, D p_{t} f(x)\right\rangle\right| \\
& =\left|\left\langle y, D p_{t} f\left(x+l_{0} y\right)\right\rangle-\left\langle y, D p_{t} f(x)\right\rangle\right|,
\end{aligned}
$$

and so by Lemma 2.5, the right side of (7) goes to 0 as $s \rightarrow 0$, uniformly for $x$ in $B$ and $y$ in a bounded subset of $B$.

Proof of Theorem 1.

$$
\begin{aligned}
\frac{\partial}{\partial t} g(x, t)= & \lim _{s \rightarrow 0} s^{-1}\{g(x, t+s)-g(x, t)\} \\
= & \lim _{s \rightarrow 0} s^{-1}\left\{p_{1-e^{-2(t+s)}} f\left(e^{-(t+s)} x\right)-p_{1-e^{-2 t}} f\left(e^{-(t+s)} x\right)\right\} \\
& +\lim _{s \rightarrow 0} s^{-1}\left\{p_{1-e^{-2 t}} f\left(e^{-(t+s)} x\right)-p_{1-e^{-2 t}} f\left(e^{-t} x\right)\right\} \\
\equiv & (\text { i })+\text { (ii), say. }
\end{aligned}
$$

Using part (b) of Lemma 2.1, we obtain (i) $=\operatorname{tr} D^{2} g(x, t)$, the limit existing uniformly for $x$ in $B$. Lemmas 2.5 and 2.6 easily yield

$$
\text { (ii) }=\left\langle e^{-t} x, e^{t} D g(x, t)\right\rangle=\lim _{s \rightarrow 0} s^{-1}\{g((1+s) x, t)-g(x, t)\} \text {, }
$$

the limit existing uniformly on bounded subsets of $B \cdot \lim _{t \downarrow 0} h(x, t)=f(x)$ uniformly on $B$ follows from Lemma 2.1 , and so $g(x, t)$ satisfies problem (2) and part (c) of Theorem 1.

Using formulas (3) and (5) it is easy to calculate that for fixed $h$ in $H$, $(x, t) \rightarrow\langle h, D g(x, t)\rangle$ is uniformly continuous on $B \times[\tau, \infty)$ for $\tau>0$. As in the proof of Lemma 2.5, for $y$ in $B$ the map $(x, t) \rightarrow\langle y, D g(x, t)\rangle$ is uniformly continuous on $B \times[\tau, \infty)$. It then follows that $(x, t) \rightarrow\langle x, D g(x, t)\rangle$ is uniformly continuous on $S \times[\tau, \infty)$ where $S$ is any bounded subset of $B$. Thus $g(x, t)$ satisfies (a) of Theorem 1.

Part (c) of Theorem 1 follows from (c) of Lemma 2.1, since

$$
D^{2} g(x, t)=\left.e^{-2 t} D^{2} p_{1-e^{-2 t}} f(y)\right|_{y=e^{-t} x} .
$$


Equation (38) of [5] gives $\left\|D^{2} p_{t} f(x)\right\|_{\mathrm{tr}} \leq c t^{-1 / 2}$ for some constant $c$ depending only on the $B$-norm, and so

$$
\left\|D^{2} g(x, t)\right\|_{t r} \leq c e^{-2 t}\left(1-e^{-2 t}\right)^{-1 / 2} \leq c e^{-2 t}\left(2 t e^{-2 t}\right)^{-1 / 2} \leq c_{1} t^{-1 / 2} e^{-t} .
$$

From (4) we obtain $|\langle x, D g(x, t)\rangle| \leq C e^{-t}\|x\|$, and so part (d) is established. This concludes the proof of Theorem 1 .

For each Borel set $\Gamma$ in $B, x$ in $B$ and $t>0$ we define

$$
o_{t}(x, \Gamma)=p_{1-e^{-2 t}}\left(e^{-t} x, \Gamma\right)=p_{1-e^{-2 t}}\left(\Gamma-e^{-t} x\right) .
$$

By Proposition 1 of $[5] o_{t}(x, \Gamma)$ and $o_{s}(y, \Gamma)$ are equivalent measures iff $s=t$ and $x-y \in H$. Since $\left\{p_{t}(x, \Gamma)\right\}$ gives the transition functions of a Markov process on $B$ with continuous sample paths and since $p_{t s}(\Gamma)=p_{t}\left(s^{-1 / 2} \Gamma\right)$, it is easily seen that $\left\{o_{t}(x, \Gamma)\right\}$ also gives the transition functions of a Markov process with sample functions given by

$$
o_{t}(x, \omega)=e^{-t} x+W_{1-e^{-2 t}}(\omega)
$$

where $W_{t}$ is the Wiener process initiating at the origin [5]. This process is an extension to abstract Wiener spaces of the velocity process of the OrnsteinUhlenbeck theory of Brownian motion with diffusion coefficient one and relaxation time one [11], [16].

The natural setting for a semigroup with transition function $\left\{p_{t}(x, \Gamma)\right\}$ is on some subspace of the bounded measurable functions with the sup norm. Property (b) of Lemma 2.1 (the uniform existence of the time derivative) enables one to show that the infinitesimal generator of such a semigroup is in a reasonable sense the closure of $\Delta[14]$. It is easily proved that the family of operators $\left\{o_{t}: t>0\right\}$, where $o_{t} f(x) \equiv \int f(y) o_{t}(x, d y)$, forms a strongly continuous contraction semigroup on the bounded uniformly continuous functions with the sup norm. However, even the functions $g(\cdot, t)$ of Theorem 1 are not necessarily in the domain of the infinitesimal generator of this semigroup, since the time derivative of $g(x, t)$ may not exist uniformly for $x$ in $B$ and since $N g$ may not be bounded. (For example, when $f(x)=\sin \langle x, y\rangle$ for some $y$ in $B^{*}$, then $f$ is bounded uniformly Lip 1, yet $\left\langle x, D o_{t} f(x)\right\rangle$ is unbounded.) This indicates that the Ornstein-Uhlenbeck velocity semigroups should be studied on spaces with norms not involving $\|\cdot\|_{\infty}$.

3. Uniqueness of solutions. In this section we impose the following condition on the $B$ norm $\|\cdot\|$. We assume that there exists an increasing sequence $\left\{Q_{n}\right\}$ of finite-dimensional projections on $B$ with $Q_{n}[B] \subset B^{*}, Q_{n}$ convergent to $I_{B}$ strongly on $B$ and $P_{n} \equiv Q_{\left.n\right|_{H}}$ convergent to $I_{H}$ strongly on $H$.

Remark 3.1. This condition has been imposed on measurable norms by Kuo [7] in work on stochastic integrals and by Piech [13] in work on parabolic equations. It is satisfied when $(H, B, i)$ is Wiener space or when $B$ is itself a 
Hilbert space (i.e. whenever $\|x\|=(A x, x)^{1 / 2}$ with $A$ a positive definite trace class operator on $H$ ) (see [13]).

Definition. $\alpha$ will denote $\inf \left\{\lambda:\left\|Q_{n} x\right\|^{2} \leq \lambda\|x\|^{2}\right.$ for all $n$ and for all $x$ in $\left.B\right\}$. (This set is nonempty by the principle of uniform boundedness.)

Definition. A real number $\lambda$ will be called an admissible $L^{2}$ exponent if $\exp \left(\lambda \alpha\|x\|^{2}\right) \in L^{2}\left(p_{1}\right)$. A real-valued function $g$ on $B$ will be said to be of exponential order if

(i) $g$ is continuous.

(ii) There exist constants $c_{1}$ and $c_{2}$ such that $|g(x)| \leq c_{1} \exp \left(c_{2}\|x\|^{2}\right)$ where $c_{2}$ is an admissible $L^{2}$ exponent. A family $\left\{g_{\alpha}\right\}$ is of exponential order uniformly for all $\alpha$ if $c_{1}$ and $c_{2}$ can be chosen independently of $\alpha$.

Comment. The identity map on $B^{*}$ may be regarded as a densely defined map of $H^{*}$ into random variables over $\left(B, p_{t}\right)$. By [4] this map extends to a representative of the normal distribution $n_{t}$ over $H$ in a unique manner. Thus elements $y$ of $H^{*}$ (or, equivalently, of $H$ ) correspond to r.v.'s $\tilde{y}^{\tilde{y}}$ on $B$. This isomorphism extends to Borel functions $f$ on $H$ which are of the form $f(x)$ $=f(P x)$ for some finite-dimensional projection $P$ on $H$. The corresponding r.v. on $B$ is denoted by $f^{\sim}$. Although this isomorphism does not extend to all continuous functions on $H$, it does extend to a reasonably large subset of them [4]. In particular $f(x) \equiv\|x\|_{1}$, with $\|\cdot\|_{1}$ a measurable seminorm on $H$, always extends to an r.v. The tilde is often omitted for notational convenience.

Remark 3.2. It is a consequence of a result of Landau and Shepp [8] that for any measurable seminorm $\|\cdot\|_{1}, \exp \left(\lambda\|x\|_{1}^{2}\right) \in L^{2}\left(p_{1}\right)$ for all $\lambda$ sufficiently near zero. For example, if $\|\cdot\|_{1}$ were given by a positive trace class operator $A$ on $H$ (see Remark 3.1) then $\lambda$ must be $<\left(4\|A\|_{L(H)}\right)^{-1}$.

Lemma 3.3. If $g$ is of exponential order then $g_{n}(x) \equiv g\left(Q_{n} x\right)$ converges to $g(x)$ in $L^{2}\left(p_{1}\right)$.

Proof. From Corollary 3 to Theorem 1 of [4], we have $g\left(F_{n} x\right)^{\sim}$ converges to $g(x)$ in probability whenever $F_{n}$ is a sequence of projections on $H$ converging strongly to $I_{H}$. Thus $g\left(P_{n} x\right)^{\sim}=g_{n}(x)$ a.e. $\rightarrow g(x)$ a.e. on $B$. But each $\left|g_{n}(x)\right|$ $\leq \exp \left(c_{2} \alpha\|x\|^{2}\right)$ and so dominated convergence gives the desired result.

Notation. $[f, g]$ will denote the $L^{2}\left(p_{1}\right)$ inner product.

Definition. Let $\delta$ be the set of cylinder functions $f$ which are twice continuously differentiable on their base spaces $P H$ and which satisfy an inequality of the form

$$
\left|D^{2} f(x)\right|_{L(H)} \leq c e^{\lambda|x|^{2}} \quad(x \in P H)
$$

for some constant $c$ and some $\lambda<1 / 4$.

Lemma 3.4. $[N f, g]=[f, N g]$ whenever $f$ and $g$ are in $\delta$.

Proof. Note that for $x$ in the base space of $f$,

$$
\begin{aligned}
|D f(x)|_{H} & \leq|D f(0)|_{H}+|x| \cdot\left|D^{2} f(\hat{x})\right| \text { where }|\hat{x}| \leq|x| \\
& \leq c_{1} e^{\lambda^{\prime}|x|} \text { for some } \lambda^{\prime} \text { in }(\lambda, 1 / 4) .
\end{aligned}
$$


Obviously $|f(x)|$ satisfies a similar inequality. Thus $f$ and its first two derivatives are in $L^{2}$ of Gauss measure of variance parameter 1 on the base space of $f$.

Case 1. Assume $f$ and $g$ have the same one-dimensional base space. Integrating by parts gives

$$
[N f, g]=(2 \pi)^{-1 / 2}\left[\left(g f^{\prime}-f g^{\prime}\right) e^{-x^{2} / 2}\right]_{-\infty}^{\infty}+[f, N g]
$$

and the evaluation at $\pm \infty$ is zero.

Case 2. Assume $f$ and $g$ may be given a common two-dimensional base space. We may write

$$
\begin{aligned}
{[N f, g]=} & (2 \pi)^{-1 / 2} \int_{-\infty}^{\infty} e^{-y^{2} / 2}\left\{(2 \pi)^{-1 / 2} \int_{-\infty}^{\infty}\left[f_{x x}-x f_{x}\right] g e^{-x^{2} / 2} d x\right\} d y \\
& +(2 \pi)^{-1 / 2} \int_{-\infty}^{\infty} e^{-x^{2} / 2}\left\{(2 \pi)^{-1 / 2} \int_{-\infty}^{\infty}\left[f_{y y}-y f_{y}\right] g e^{-y^{2} / 2} d y\right\} d x
\end{aligned}
$$

It is easy to see that Case 1 applies to the inside integrals; and so $[N f, g]$ $=[f, N g]$.

General case. Proof obvious from Case 2.

Lemma 3.5. If $\left\|D^{2} g\right\|_{t r}$ is of exponential order, and if $g_{n}$ is defined as in Lemma 3.3 then $g_{n}$ is in $\delta$.

Proof. Since $D^{2} g_{n}$ is of finite rank $\left\|D^{2} g_{n}\right\|_{\mathrm{tr}}$ is equivalent to $\left|D^{2} g_{n}\right|_{L(H)}$. Now $\left|D^{2} g_{n}\left(P_{n} x\right)\right|_{L(H)} \leq c_{1} \exp \left(c_{2}\left\|P_{n} x\right\|^{2}\right)$ which is in $L^{2}$ of Gauss measure on $P_{n} H$. We claim that this implies that $c_{2}\left\|P_{n} x\right\|^{2} \leq \lambda\left|P_{n} x\right|^{2}$ for some $\lambda<1 / 4$. For since $\|\cdot\|$ is continuous on $H$ it is easily seen that

$$
\int_{R^{1}} \exp \left\{2 c_{2}\left\|x_{1}+y\right\|^{2}\right\} \exp \left\{-\left|x_{1}\right|^{2} / 2\right\} d x_{1}
$$

is a continuous function of $y$ on $P_{n} H$ modulo the span of $x_{1}$. Thus by Fubini's theorem, (8) is finite for all $y$, and in particular for $y=0$. Since $\|\cdot\|$ and $|\cdot|$ are scalar multiples in one dimension, we must have $2 c_{2}\left\|x_{1}\right\|^{2}=\mu_{1}\left|x_{1}\right|^{2}$ where $\mu_{1}<\frac{1}{2}$. If $P_{n} x=x_{1}+\cdots+x_{n}$ with $x_{i}$ orthogonal (in $H$ ) to $x_{j}$, then we see that each $\left\|x_{i}\right\|^{2}<\frac{1}{4}\left|x_{i}\right|^{2}$. Thus $g_{n} \in \delta$.

Lemma 3.6. If $g: B \rightarrow R^{1}$ with $g, N g$ and $\left\|D^{2} g\right\|_{t r}$ each of exponential order, then $N g_{n} \rightarrow N g$ in $L^{2}\left(p_{1}\right)$.

Proof. A straightforward calculation yields

By Lemma 3.3, $N g\left(Q_{n} x\right) \rightarrow N g(x)$ in $L^{2}$. It is easily seen that the right side of (9) $\rightarrow 0$ a.e. But the right side of (9) is dominated by $\left\|D^{2} g\left(Q_{n} x\right)\right\|_{\mathrm{tr}}$ which in turn is dominated by a fixed $L^{2}$ function. Dominated convergence gives the desired result.

Theorem 2. If $u: B \times[0, \infty) \rightarrow R^{1}$ satisfies

(i) $u(\cdot, t)$ is of exponential order uniformly for $t \in[0, \infty) ;|N u(x, t)|$ and $\left\|D^{2} u(x, t)\right\|_{\mathrm{tr}}$ are each dominated by $c_{t} \exp \left(c_{2}\|x\|^{2}\right)$ where $c_{2}$ is an admissible $L^{2}$ exponent and $c_{t}$ is integrable on $[0, \tau]$ for each $\tau>0$. 
(ii) $N u(x, \cdot)$ is continuous on $(0, \infty)$ for each $x$.

(iii) $N u=\partial u / \partial t$.

(iv) $u(x, t) \rightarrow 0$ as $t \rightarrow 0$ uniformly for $x$ in $B$.

Then $u(x, t) \equiv 0$.

Lemma 3.7. If $f$ is bounded uniformly Lip 1 on $B$. Then $o_{t} f(x)$ satisfies hypotheses (i)-(iii) of Theorem 2.

Proof. Part (d) of Theorem 1.

Proof of Theorem 2 (method of Ito [6]). Let $u$ satisfy the hypotheses of the theorem and let $f$ be bounded and uniformly Lip 1 on $B$.

We first note that $o_{t} f_{n}(x) \equiv o_{t}\left(f \circ Q_{n}\right)(x)=\left(o_{t} f\right)\left(Q_{n} x\right) \equiv\left(o_{t} f\right)_{n}(x)$. Thus for $0<t<a$, Lemmas 3.3, 3.5 and 3.7 give

$$
\begin{aligned}
{\left[N u, o_{a-t} f\right] } & =\lim _{n}\left[(N u)_{n}, o_{a-t} f_{n}\right]=\lim _{n}\left[N u_{n}, o_{a-t} f_{n}\right], \\
\left.\|(N u)_{n}, o_{a-t} f_{n}\right] & \leq c \cdot c_{t}, \\
\left.\|(N u)_{n}, o_{a-t} f_{n}\right] & =\|\left[u_{n}, N o_{a-t} f_{n}\right] \mid \leq c t^{-1 / 2} .
\end{aligned}
$$

Thus we may write

$$
\begin{aligned}
\int_{0}^{a}\left[N u, o_{a-t} f\right] & d t=\lim _{n} \int_{0}^{a}\left[(N u)_{n}, o_{a-t} f_{n}\right] d t=\lim _{n} \int_{0}^{a}\left[\frac{\partial}{\partial t} u_{n}, o_{a-t} f_{n}\right] d t \\
= & \lim _{n} \int_{B} \int_{0}^{a} \frac{\partial}{\partial t} u_{n}(x, t) o_{a-t} f_{n}(x) d t p_{1}(d x) \\
& =\lim _{n} \int_{B} u_{n}(x, a) f_{n}(x) p_{1}(d x)-\lim _{n} \int_{B} \int_{0}^{a} u_{n}(x, t) \frac{\partial}{\partial t} o_{a-t} f_{n}(x) d t p_{1}(d x) \\
& =[u(\cdot, a), f]-\lim _{n} \int_{0}^{a} \int_{B} u_{n}(x, t) \frac{\partial}{\partial t} o_{a-t} f_{n}(x) p_{1}(d x) d t \\
& =[u(\cdot, a), f]+\lim _{n} \int_{0}^{a} \int_{B} u_{n}(x, t) N o_{a-t} f_{n}(x) p_{1}(d x) d t \\
& =[u(\cdot, a), f]+\lim _{n} \int_{0}^{a}\left[N u_{n}, o_{a-t} f_{n}\right] d t \\
& =[u(\cdot, a), f]+\int_{0}^{a}\left[N u, o_{a-t} f\right] d t .
\end{aligned}
$$

Hence $[u(\cdot, a), f]=0$ for all $f$ which are bounded and uniformly Lip 1 on $B$. But such $f$ 's are dense in $L^{2}\left(p_{1}\right)$. Since $u$ is continuous, we have $u(x, t) \equiv 0$.

Corollary 3.8 (Uniqueness of solutions). If $u$ satisfies hypotheses (i)-(iii) of Theorem 2 , and $u(x, t) \rightarrow f(x)$ uniformly for $x$ in $B$ where $f$ is bounded uniformly Lip 1 , then $u(x, t) \equiv o_{t} f(x)$.

Remark 3.9. The symmetry of $N$ on a suitable subspace of $L^{2}\left(p_{1}\right)$ is crucial for the preceding proof. The Laplacian $\Delta$ fails to possess such a symmetry property.

Remark 3.10. For the Cauchy problem for a parabolic equation in $R^{n}$ uniqueness of solutions of exponential order is usually obtained only on finite time intervals. Uniqueness for all time would usually require a hypothesis like space boundedness. In our case we obtain uniqueness for all time mainly because the transition measures actually only run through Wiener measures with variance 
parameters from zero to one, rather than from zero to infinity as in the general finite-dimensional case.

4. A change of second order coefficients. The coefficients of the second order part of the operator $N$ may be modified by a technique described in [14]. We can thus obtain the following

Theorem 3. Let $A: H \rightarrow H$ be symmetric, bounded below by $\varepsilon I(\varepsilon>0)$, and let $I-A$ be of Hilbert-Schmidt class. Then the results of Theorems 1 and 2 hold when $N$ is replaced by

$$
N_{A} f(x) \equiv \operatorname{tr}\left[A D^{2} f(x)\right]-\lim _{s \rightarrow 0} \frac{f((1+s) x)-f(x)}{s}
$$

and $o_{t}(x, \Gamma)$ replaced by

$$
o_{t}^{A}(x, \Gamma)=p_{1-e^{-2 t}}^{A}\left(e^{-t} x, \Gamma\right)
$$

where

$$
p_{t}^{A}(x, \Gamma)=p_{t} \circ A^{-1 / 2}(\Gamma-x) .
$$

\section{REFERENCES}

1. V. Goodman, $A$ divergence theorem for Hilbert space, Trans. Amer. Math. Soc. 164 (1972), 411-426. MR 45 \#7469.

2.—, Quasi-differentiable functions on Banach spaces, Proc. Amer. Math. Soc. 30 (1971), 367-370. MR 44 \#2035.

3. L. Gross, Measurable functions on Hilbert space, Trans. Amer. Math. Soc. 105 (1962), 372-390. MR 26 \#5121.

4. - Abstract Wiener spaces, Proc. Fifth Berkeley Sympos. Math. Statist. and Probability (Berkeley, Calif., 1965/66), vol. II: Contributions to Probability Theory, Part 1, Univ. California Press, Berkeley, Calif., 1967, pp. 31-42. MR 35 \#3027.

5.—, Potential theory on Hilbert space, J. Functional Analysis 1 (1967), 123-181. MR 37 \#331.

6. S. Itô, The fundamental solution of the parabolic equation in a differentiable manifold, Osaka Math. J. 5 (1953), 75-92. MR 15, 36.

7. H. - H. Kuo, Stochastic integrals in abstract Wiener space, Pacific J. Math. 41 (1972), 469-483.

8. H. J. Landau and L. A. Shepp, On the supremum of a Gaussian process, Sankhyā, Ser. A 32 (1970), 369-378. MR 44 \#3381.

9. H. P. McKean, Geometry of differential space, Ann. of Probability 1 (1973), 197-206.

10. E. Nelson, An existence theorem for second order parabolic equations, Trans. Amer. Math. Soc. 88 (1958), 414-429. MR 20 \# 1844.

11. E. Nelson, Dynamical theories of Brownian motion, Princeton Univ. Press, Princeton, N. J., 1967. MR $35 \# 5001$.

12.—, Quantum fields and Markoff fields (preprint).

13. M. A. Piech, A fundamental solution of the parabolic equation on Hilbert space. II: The semigroup property, Trans. Amer. Math. Soc. 150 (1970), 257-286. MR 43 \#3847.

14._- Differential equations on abstract Wiener space, Pacific J. Math. 43 (1972), 465-473.

15. I. Segal, Construction of non-linear local quantum processes. I, Ann. of Math. (2) 92 (1970), 462-481. MR 42 \#7187.

16. G. E. Uhlenbeck and L. S. Ornstein, On the theory of Brownian motion, Phys. Rev. 36 (1930), 823-841.

17. Y. Umemura, On the infinite dimensional Laplacian operator, J. Math. Kyoto Univ. 4 (1964/65), 477-492. MR 31 \#5102.

Department of Mathematics, State University of New York at Buffalo, Amherst, New YORK 14226 